\title{
ADHERENCIA DEL PACIENTE A LA DIÁLISIS PERITONEAL
}

\author{
Ana Julia Carrillo Algarra*, Marcela Marín Rincón**, Linda Lenny Ortiz Ruiz**
}

\begin{abstract}
Resumen
Objetivo: explorar los factores que influyen en la adherencia de los pacientes adultos a la diálisis peritoneal, la medición de la misma y las consecuencias de la no adherencia reportadas en la literatura. Metodología: consulta a los descriptores en ciencias de la salud (DeCS), adherencia, insuficiencia renal terminal, diálisis peritoneal y autocuidado, con el buscador booleano AND. Se encontraron 50 artículos, 40 en español, nueve en inglés y uno en portugués, publicados entre 1995 y 2013; se utilizó la metodología del Critical Appraisal Skills Programme España (CASPe) y la guía teórico-práctica sobre construcción de estados del arte de Consuelo Hoyos. Resultados y conclusiones: los factores que influyen están relacionados con características individuales, aspectos sociales, equipo terapéutico y redes de apoyo. Se han utilizado parámetros clínicos (ganancia de peso interdialítico y episodios de peritonitis) y bioquímicos (hipercalemia, elevación del nitrógeno ureico y creatinina) como indicadores para medir la adherencia, además de las pruebas de Morisky Green, la escala de adherencia terapéutica para pacientes con enfermedades crónicas basada en comportamientos explícitos (EAT) y la escala analógica visual (EAV). En enfermería la adherencia es relevante y está incluida en las taxonomías diagnóstica, de intervención y de medición de resultados.
\end{abstract}

Palabras clave: adherencia, adhesión, insuficiencia renal terminal, diálisis peritoneal, autocuidado.

Abreviaturas: ERC, enfermedad renal crónica; DP, diálisis peritoneal.

\section{ADHERENCE TO HEMODIALYSIS}

\section{Abstract}

Objective: to explore scientific literature regarding factors influencing the adherence to hemodialysis emphasizing on personality and internal control. Design and Methodology: a search of articles in MdConsult, Ovid, Ebsco Host and UpToDate databases. Those which contained at least two of the key words were selected. Results and Conclusions: personality can affect adherence in two ways, the first is related to behavior and the second to each person's perception of the world and how he/she relates to it. Internal control influences adherence behavior to treatment, hence, the various internal and external factors which affect the individual must be assessed.

Key words: hemodialysis, adherence, personality, internal control.

Fecha recibido: mayo I4 de 2013 - Fecha aceptado: marzo II de 2014

* Enfermera. Profesor Titular Facultad de Enfermería de la Fundación Universitaria de Ciencias de la Salud. Bogotá DC, Colombia.
** Estudiantes de la especialización en enfermería nefrológica y urológica. Fundación Universitaria de Ciencias de la Salud. Bogotá DC, Colombia. 


\section{Introduc ción}

La insuficiencia renal terminal es una situación clínica no transmisible, progresiva, de incidencia y prevalencia elevadas ${ }^{1}$ en la que ocurre pérdida irreversible de la función renal, por tanto el paciente depende en forma permanente de un tratamiento sustitutivo que puede ser hemodiálisis, diálisis peritoneal o trasplante. ${ }^{2}$

El continente latinoamericano está en particular riesgo debido a mayor prevalencia de enfermedades crónicas en la actualidad, falta de políticas sanitarias que promuevan la salud renal y la disparidad de acceso a la salud en distintas regiones. ${ }^{3}$ En Colombia por ejemplo, en el 2007 la prevalencia de enfermedad renal crónica (ERC) fue de 403.16 personas por millón de habitantes, siendo las etiologías más frecuentes diabetes mellitus e hipertensión arterial. ${ }^{4}$ En 2005 el $40 \%$ de los pacientes en tratamiento se encontraban en diálisis peritoneal y el $60 \%$ en hemodiálisis ${ }^{5}$, en 2008 se presentaron 21.572 casos de ERC estadio 5, ${ }^{6}$ y para 2010 la prevalencia bruta de ERC fue $0.87 \%$ cifra considerada baja comparada con otros países, lo que podría deberse a que los estadios tempranos de la enfermedad son usualmente asintomáticos y con frecuencia no detectados y subestimados, con el consiguiente subregistro de información. En el mismo año el $5.27 \%$ de los pacientes con ERC estaban clasificados en estadio $5 .^{7}$

En éste contexto es muy importante la adherencia que la persona tenga al tratamiento, la cual es definida por La Organización Mundial de la Salud (OMS) como la magnitud con que el paciente sigue las instrucciones médicas, retomando a Haynes y Sackett quienes dicen que es "la medida en que la conducta del paciente coincide con las indicaciones dadas por su médico en relación con la toma de medicación, el seguimiento de una dieta o la modificación del estilo de vida". ${ }^{8}$ En algunos estudios se asume adherencia como sinónimo de cumplimiento, pero la diferencia principal entre estos dos conceptos radica en que la adherencia requiere conformidad del paciente respecto a las recomendaciones y buena comunicación entre el cuidador formal y el usuario como un requisito esencial para una práctica clínica efectiva. ${ }^{9}$
Las personas con afecciones crónicas experimentan cambios en su modo de vida y la mayoría de la atención necesaria se basa en autocuidado, pero se ha detectado que el incumplimiento terapéutico alcanza un $45 \%$, porcentaje que se incrementa "cuando los tratamientos generan cambios en los hábitos de vida e invaden diversas áreas (laboral, social, familiar). ${ }^{10}$ Todo lo anterior, sumado al número de personas que se encuentran en diálisis peritoneal, los diversos factores que influyen en el éxito de la misma y sobre todo que es un procedimiento realizado por el mismo paciente o es ejecutado por un cuidador no formal, la mayoría de las veces educados por enfermería, despertó el interés de las investigadoras para explorar que dice la literatura científica sobre los factores que influyen en la adherencia al tratamiento en pacientes en diálisis peritoneal, diferentes formas de medir la misma y consecuencias de la no adherencia.

\section{Metodología}

Se realizó la búsqueda en las bases de datos MdConsult, Ovid, Ebsco Host, UpToDate; las palabras claves se combinaron con el buscador booleano AND y se incluyeron los artículos que contenían por lo menos dos de los términos utilizados. Se encontraron 200 artículos, de los cuales una vez revisado el resumen, se seleccionaron 40 en español, 9 en inglés y 1 en portugués, escritos en el periodo comprendido entre 1995 y 2013. A los artículos seleccionados se les aplicó la metodología del Critical Appraisal Skills Programme España (CASPe) ${ }^{11}$, se tomó como referencia disciplinar la teoría de Dorothea Orem ${ }^{12}$ y para el análisis la guía teórico-práctica sobre construcción de estados del arte de Consuelo Hoyos. ${ }^{13}$

\section{Resultados}

La literatura revisada reporta diferentes definiciones de adherencia, entre ellas la adoptada por el grupo de adherencia terapéutica de la OMS (2004) que fusiona las definiciones de Haynes y Sackett "grado en que el comportamiento de una persona -tomar el medicamento, seguir un régimen alimentario y ejecutar cambios del modo de vida- se corresponde con las recomendaciones acordadas con un prestador de asis- 
tencia sanitaria". ${ }^{9,14}$ Teniendo en cuenta que enfermería cuida al ser humano en sus diferentes dimensiones, se retomaron definiciones más amplias como la de Soria y cols. (2009) quienes plantean que "...la adherencia en el ámbito psicológico se refiere al conjunto de comportamientos efectivos para el cumplimiento de las prescripciones médicas que conllevan al control de la enfermedad. Este conjunto de comportamientos explícitos tendría que contemplar si el paciente ingiere los medicamentos y alimentos prescritos, si sus conductas son efectivas para mejorar su salud y su creencia de si considera que lo que hace es eficaz para controlar su enfermedad... ${ }^{15}$ Esta incluye no solo aspectos biológicos sino también del comportamiento y capacidad de autocuidado de la persona.

Otro concepto importante es el de diálisis peritoneal, definida como un proceso de difusión selectiva a través de una membrana, que se utiliza para la separación de moléculas de diferente tamaño a través del peritoneo, pero más allá de la definición del procedimiento el grupo investigador encontró que también es concebida como "una técnica domiciliaria de tratamiento sustitutivo de la función renal"16,17, que permite al paciente realizar su tratamiento en casa, tener independencia, continuar sus actividades laborales, tener un mayor bienestar físico y mental y por lo tanto disfrutar de una mejor calidad de vida; para lograr estos beneficios es importante la adherencia al tratamiento tanto del paciente como del familiar que colabora en el procedimiento.

Desde el punto de vista de los aspectos formales de los artículos, la fecha de inicio de la búsqueda se estableció en 1995, pero el 80\% fueron publicados entre 2007 y 2013, mostrando un reciente interés por investigar sobre el tema, que lleva a preguntarse qué influencia tuvo la discusión sobre enfermedades crónicas realizada en la 59a asamblea mundial de la salud de $2006^{18}$ y el cambio en el perfil epidemiológico en los países en vía de desarrollo. El mayor número de artículos se encontró en Europa, en especial España (50\%), seguido de Norteamérica, haciendo énfasis en Estados Unidos (30\%) y Suramérica (10\%), y con menor porcentaje el continente asiático que corresponde al $2 \%$, lo cual es coherente con el perfil epidemiológico que demuestra que las enfermedades crónicas tienen mayor prevalencia en los países desarrollados y el comportamiento de la enfermedad renal en el mundo. Además, este resultado tiene la influencia de los idiomas elegidos para la búsqueda. Llama la atención que en Colombia no se publiquen más estudios al respecto si se tiene en cuenta que la guía basada en la evidencia para la atención de la enfermedad renal crónica (2005) refiere que "actualmente el paciente con ERC, que tenga función renal residual, la primera opción de tratamiento dialítico debe ser la terapia de diálisis peritoneal". ${ }^{19}$

La clasificación de los artículos por objetivos reportó que el $36 \%$ de los mismos corresponde a factores que influyen en forma directa en la adherencia a la DP, seguido del $24 \%$ que hacen referencia a las consecuencias o efectos de la no adherencia y en menores porcentajes se observó que el 16\%, 14\% y 10\%, dan respuesta a interrogantes relacionados con la eficacia, evaluación e identificación de redes de apoyo que actúan en la permanencia y adherencia de los pacientes con ERC que se encuentran en tratamiento de DP y los parámetros que permiten identificar la adherencia al tratamiento (Tabla 1).

\begin{tabular}{|c|c|c|c|}
\hline & Objetivos & $\mathbf{N}$ & $\%$ \\
\hline I. & $\begin{array}{l}\text { Identificar los factores que influyen en la adheren- } \\
\text { cia al tratamiento }\end{array}$ & 18 & 36 \\
\hline 2. & $\begin{array}{l}\text { Describir las consecuencias o efectos de la no } \\
\text { adherencia al tratamiento }\end{array}$ & 12 & 24 \\
\hline 3. & $\begin{array}{l}\text { Explorar la eficacia o efectividad de las interven- } \\
\text { ciones del equipo de salud en la adherencia al } \\
\text { tratamiento }\end{array}$ & 8 & 16 \\
\hline 4. & $\begin{array}{l}\text { Identificar el efecto de la influencia de las redes de } \\
\text { apoyo en la adherencia al tratamiento }\end{array}$ & 7 & 14 \\
\hline 5. & $\begin{array}{l}\text { Describir y evaluar los parámetros que permiten } \\
\text { identificar la adherencia al tratamiento }\end{array}$ & 5 & 10 \\
\hline \multicolumn{2}{|c|}{ Total } & 50 & 100 \\
\hline
\end{tabular}

Con base en el análisis de los objetivos de los artículos, emergieron las categorías que facilitan la comprensión de los factores que intervienen en la adherencia del paciente en forma directa o indirecta como la influencia del equipo de salud, las consecuencias de la no adherencia y las diferentes formas de medirla. 


\section{Factores que influyen en la adherencia a la diálisis peritoneal}

Existen muchos factores que influyen en esta conducta, desde las diferentes dimensiones del ser humano. La literatura reporta falta de motivación, desconocimiento, baja autoestima y depresión ${ }^{20}$, otros artículos hacen énfasis en el estado de ánimo, pues ante los cambios que surgen en esta nueva forma de vivir, las personas en diálisis pueden desarrollar cambios en su comportamiento. ${ }^{21}$ Según Malheiro (2012) es necesario comprender la calidad de vida de los pacientes con ERC teniendo en cuenta que es una enfermedad que genera alteraciones físicas, psicológicas y en sus roles cotidianos y sociales. ${ }^{22-24}$ Todos estos cambios se ven reflejados en una peor percepción de la calidad de vida con respecto a la población general ${ }^{23,24}$, pues es lo que permite a los cuidadores establecer la comodidad de sus familiares.

La familia desempeña una labor importante en la adherencia al tratamiento de DP, porque permite a los pacientes sentirse seguros del manejo que se les da en casa y disminuir el riesgo de complicaciones en el procedimiento; se establece que existe adherencia cuando el paciente y su familiar logran un buen manejo de la práctica, le dan importancia al mantenerse sano y muestran menos factores estresantes ante la realización de los recambios. ${ }^{25}$

Con respecto al tipo de terapia, García y cols. (2008) en un estudio comparativo entre 30 pacientes en hemodiálisis y 31 en DP encontraron que los últimos informaron niveles superiores de adhesión y mejor calidad de vida relacionada con la salud en función física y dolor corporal ${ }^{26}$, resultado coherente con otros estudios que reportan que la hemodiálisis hace dependiente al paciente mas no adherente ${ }^{27}$, la diálisis se convierte en el eje de su vida implicando grandes restricciones que están dispuestos a seguir en la mayoría de los casos, en comparación con los que están en $\mathrm{DP}^{28}$ quienes tienen más flexibilidad de horarios y menos restricciones para los desplazamientos, logrando alcanzar una mayor compatibilidad con otras responsabilidades. Se considera que el nivel de autosuficiencia y la capacidad de autocuidado es superior en DP, a pesar de las ventajas e inconvenientes de ambos tratamientos, sin que haya un acuerdo completo respecto a que modalidad más beneficiosa. ${ }^{29}$

Un factor que mejora la adherencia del paciente a la DP es la educación acerca del autocuidado, porque aumenta la participación activa en su tratamiento, para ello es necesario conocer los aspectos personales y del entorno, que permiten la realización de la DP continua ambulatoria (DPCA) en el hogar del paciente y contar con un miembro de la familia con disposición de tiempo para el entrenamiento, que le sirva de apoyo sin disminuir la independencia y la participación activa del paciente en el cuidado. ${ }^{26}$ Sin embargo, para la realización del tratamiento, no solo basta con tener el conocimiento, también son necesarias ciertas condiciones físicas del entorno para garantizar la seguridad del paciente y evitar la contaminación del catéter de diálisis, asegurar su funcionalidad y la efectividad de la terapia de manera continua y segura. Por otra parte en su tesis doctoral Vélez (2010) reportó que tienen mayor adherencia las mujeres, las personas con mayor conocimiento respecto a la enfermedad y quienes tuvieron un informe positivo respecto al control personal definido como "creencias del paciente sobre su habilidad personal para controlar los síntomas". Como un aspecto que disminuye la adherencia encontró la creencia de no contar con la habilidad para seguir el tratamiento establecido por el médico. ${ }^{30}$

\section{El equipo de salud y la adherencia al tratamiento}

Teniendo en cuenta que según la asociación para la lucha contra las enfermedades del riñón (ALCER, 2010) la mayoría de los nefrólogos cree que la DP domiciliaria es la mejor opción de tratamiento sustitutivo inicial debido a factores como mantener la función renal residual, con las ventajas terapéuticas para la supervivencia del paciente en los primeros años y permitir el paso al trasplante renal sin alterar demasiado sus condiciones de vida Además el paciente puede mantener su vida laboral y personal, mayor libertad, independencia y más intimidad en su tratamiento. ${ }^{31}$ Es importante estudiar el papel del personal de salud sobre la adherencia. En la actualidad hay estudios que muestran que una relación de colaboración, puede contribuir a mejorar la adherencia al tratamiento en 
enfermedades crónicas ${ }^{32}$, resaltando el papel de la calidad de la comunicación del equipo de salud con el paciente y la satisfacción de éste último con la prestación de los servicios. ${ }^{10}$

En el trabajo Adherencia a los tratamientos a largo plazo (2005) se detectaron como barreras vinculadas los factores del sistema de salud, y con respecto a los proveedores de atención, la falta de conciencia y conocimiento acerca de la adherencia terapéutica, así como de herramientas clínicas que ayuden a los profesionales de la salud a evaluar e intervenir en los problemas de adherencia. También se encontró un déficit de herramientas que ayuden a las personas en condición de cronicidad a desarrollar comportamientos adaptativos saludables o modificar los problemáticos. Otro aspecto importante es la falta de comunicación entre los pacientes y los profesionales de la salud. ${ }^{9}$

Enfermería en los programas de DP es quizás uno de los pilares más importantes en la adherencia al tratamiento, Montenegro y cols. (2009) describen dentro del perfil de la enfermera "es muy importante disponer de buena capacidad docente y de comunicación, ser flexible, tener iniciativa e imaginación"33, características indispensables en la práctica diaria. Su papel es determinante en el éxito de los programas y en la permanencia de los pacientes que requieren de estas terapias; la consulta de enfermería facilita los procesos sistematizados y planificados, sustentados en el conocimiento científico y legal, donde el objetivo principal es promover la cultura del autocuidado con la participación del paciente de manera efectiva, segura y eficaz, por medio de estrategias de educación en salud, investigación comunitaria y pública, y servicio asistencial y administrativo. Establece vínculos de apoyo y confianza enfermera-paciente, lo cual se refleja en los indicadores de satisfacción del usuario, disminución de frecuencia de infecciones y mejoría de la calidad de vida de los pacientes que repercutirán en una mayor supervivencia y calidad de vida. ${ }^{34}$

En concordancia con los conceptos anteriores la Nursing Diagnoses: Definitions and Classification (NANDA) 2009-2011 definió los diagnósticos de enfermería sobre gestión ineficaz del régimen terapéutico familiar y de la propia salud incluyendo los diagnósticos encaminados a promover una salud mental emocional óptima, un funcionamiento social satisfactorio y en forma específica el diagnóstico de incumplimiento, definido como la conducta de una persona o de un cuidador que no coincide con el plan terapéutico o de promoción de la salud acordado entre la persona y un profesional. ${ }^{35}$ Con el fin de mejorar la situación del paciente plantea como resultado esperado, generar en la persona una conducta de cumplimiento definida como "las acciones personales basadas en el asesoramiento profesional para promocionar el bienestar, la recuperación y la rehabilitación." Para ello propone como intervenciones enfermeras establecer un acuerdo con el paciente y construir objetivos comunes con el fin de modificar la conducta. ${ }^{36}$

Los conocimientos proporcionados por el personal de salud sobre la responsabilidad en el tratamiento, la higiene personal y las características de la vivienda de los pacientes suelen incumplirse y llevar a continuas infecciones. Por ello surge la necesidad de reforzar la capacitación sobre el autocuidado en aras de mantener su salud y prevenir riesgos. ${ }^{37}$ Diversos estudios han mostrado la utilidad de intervenciones informativas, educativas, de apoyo familiar ${ }^{38}$ y dinámicas de grupo e intervenciones de tipo conductual, para incrementar la adherencia del paciente. ${ }^{14} \mathrm{El}$ personal de enfermería en DP tiene como función fundamental que el paciente y el cuidador no formal aprendan procedimientos, actitudes y conceptos básicos para el correcto autotratamiento. ${ }^{39}$ El éxito de la DP se basa en la buena formación del paciente para la realización de la terapia, así como una relación fluida paciente-enfermera. ${ }^{16}$

Enfermería es consciente de las consecuencias de la no adherencia y su costo elevado para el paciente, la comunidad y el sistema de atención de salud, está familiarizada con las frustraciones por los fracasos y la insatisfacción del paciente que acompañan a la adherencia deficiente. ${ }^{40}$ Dado que gran parte del tratamiento para los procesos crónicos tiene lugar en el hogar y entornos comunitarios, constituye un enlace y apoyo mediante visitas domiciliarias y llamadas telefónicas. Algunas de las estrategias que ha desarrollado para mejorar la adherencia según la OMS (2004) son: 
- Evaluar el grado de adherencia mediante preguntas no amenazantes e indagar acerca de los efectos colaterales de la medicación y su efecto sobre la calidad de vida del paciente.

- Educar a los pacientes y cuidadores no formales acerca de la enfermedad, la importancia de la adherencia, modo en que ayudará el tratamiento, efectos colaterales posibles y cómo afrontarlos.

- Sugerir señales y recordatorios e integrar los momentos de medicación con los hábitos diarios, usar pastilleros, cronómetros, alarmas y localizadores.

- Recompensar y reforzar el comportamiento de adherencia, por ejemplo con esquemas y gráficos que expongan la repercusión de la medicación sobre los marcadores clínicos de la enfermedad.

- Motivar al paciente para que cultive relaciones terapéuticas con los profesionales de la salud y converse con los compañeros y miembros de la familia.

- Asegurar que se siguen los regímenes de tratamiento y administrar los medicamentos y demás tratamientos. ${ }^{9}$

- El Consejo Internacional de enfermeras calcula que hay doce millones de enfermeras en el mundo quienes por sus características de formación y la cercanía al paciente y cuidador informal pueden constituir un apoyo para los pacientes con enfermedades crónicas. ${ }^{9}$

La literatura revisada reporta que fortalecer el autocuidado en los pacientes con enfermedades crónicas puede mejorar sus condiciones de salud, su calidad de vida, la de su familia y en forma indirecta disminuir los costos para el sistema. Las estrategias incluyen fortalecer el aprendizaje y la autorregulación, con relación a la capacidad de autocuidado, Carrillo y Díaz (2013) encontraron que en pacientes en DP la media aritmética reportada por la escala Appraisal of Selfcare Agency Scale (ASA), fue de 76.4 puntos (DE: 5.5), cuando se relaciona este dato con los hallazgos del test de Morisky-Green y los obtenidos por Rivera y Díaz, en pacientes con hipertensión arterial, se ratifica la capacidad de la escala ASA para medir las acciones de autocuidado que tienen las personas que sufren una enfermedad crónica. ${ }^{41}$

\section{Consecuencias o efectos de la no adherencia}

La no adherencia incluye aspectos como omitir recambios, no tomar medicamentos prescritos, no respetar los intervalos, dosis o frecuencias, y no ajustarse a los cambios en la dieta. Sin embargo, los términos cumplimiento o incumplimiento siguen siendo los más utilizados en la práctica médica; las tasas de cumplimiento descritas en enfermedades crónicas varían del $0 \%$ al $90 \%$, situándose la media en un $50 \%{ }^{4}$, de este porcentaje es importante tener en cuenta que los adolescentes son los que más influyen en este tipo de incumplimientos por múltiples factores que pueden estar relacionados con la deficiente adhesión terapéutica farmacológica, los efectos adversos, el tiempo, una escasa educación sanitaria, una pobre comunicación entre médico/enfermera/paciente, y el entorno pueden llevar a los pacientes a interrumpir sus tratamientos o hacerlos deficientes. ${ }^{42}$

El ingreso en terapias dialíticas en condiciones inadecuadas demanda incomodidad y sufrimiento a las personas con ERC, incrementa costos al sistema de salud y trabajo a enfermería. Pacheco (1997) evidenció que el déficit de conocimiento sobre las consecuencias de la hipertensión arterial y diabetes asociados con la ERC, y las dudas sobre esa enfermedad pueden interferir en la adhesión al tratamiento y comprometer su calidad de vida. ${ }^{43}$ La adherencia tiene gran trascendencia en las enfermedades crónicas, porque el incumplimiento lleva a un tratamiento ineficaz, produce aumento en la morbimortalidad y aumenta los costos del sistema de salud. ${ }^{9}$ Las infecciones sin duda alguna son uno de los problemas más evidentes de la no adherencia de los pacientes en DP, casi siempre por bacterias gram negativas que colonizan el orificio de salida y migran causando peritonitis. ${ }^{44}$ Como la mayoría de los casos no presentan un solo episodio sino varios, esto conlleva al mal funcionamiento de la membrana porque la deteriora, con la consecuente disminución en la capacidad de ultrafiltración de toxinas y desechos. ${ }^{45.46}$ Esta es una de las causas más 
frecuentes por la cual los pacientes en DP abandonan el tratamiento, por sus efectos clínicos o porque se convierte en un factor estresante para continuar.

\section{Medición de la adherencia al tratamiento}

El personal de salud ha tratado de medir la adherencia al tratamiento en DP con diferentes métodos como el recuento de comprimidos, la revisión de los armarios de medicamentos, pruebas de laboratorio en especial los indicadores de potasio y sodio (electrolitos), así como el control de peso como pruebas útiles para conocer la adherencia del paciente al régimen de alimentación específico. Otras formas son la medición de los conocimientos sobre la enfermedad, la técnica y parámetros de procedimientos, las entrevistas, el reconocimiento del grado de depresión y ansiedad ${ }^{25}$, y con base en ello ha iniciado la creación de protocolos, guías y cuestionarios que tienen como objetivo medir los estilos de vida específicos para este tipo de pacientes y para el cuidador, quien cumple un papel fundamental en el cuidado del paciente y en ocasiones se sobrecarga de responsabilidades y factores que pueden llegar a alterar su propio estilo de vida.

La evaluación exacta de la adherencia terapéutica es necesaria para la planeación de tratamientos efectivos, eficientes, que logren cambios en los resultados de salud atribuibles al régimen recomendado y con base en ello tomar las decisiones para cambiar las recomendaciones, los medicamentos o el estilo de comunicación. Sin embargo, no existe "patrón de oro" alguno para medir el comportamiento de adherencia terapéutica y en la bibliografía se ha informado el empleo de una variedad de estrategias. ${ }^{42}$ Por ejemplo, Nau y cols. (2008) reportan la escala analógica visual (EAV) como un medida relativamente nueva para los estudios de adherencia que por sus características podría ser utilizada en enfermedades crónicas ${ }^{47}$ y Soria y cols. (2009) publicaron la "escala de adherencia terapéutica para pacientes con enfermedades crónicas, basada en comportamientos explícitos" (EAT) a la cual le realizaron análisis métricos y relacionados con las expectativas teóricas, los cuales reportaron que la manera como están redactados los ítems y la opción de repuesta es clara, y el análisis factorial muestra "excelentes propiedades psicométricas del instrumento". Desde el punto de vista de las expectativas teóricas, evalúa los aspectos psicológicos, los conductuales que favorecen o no la adherencia y la autoeficacia. Una ventaja es que ha sido diseñada pensando en que pueda ser útil para evaluar la adherencia de personas con afecciones crónicas sin estar dirigida a una enfermedad específica". ${ }^{15}$ Otro instrumento que ha sido utilizado es el cuestionario propuesto por Martín-Bayarre-Grau (2008) para medir adherencia al tratamiento de hipertensión arterial, considerada como una de las principales causas de ERC. ${ }^{48}$ Sin embargo, García y cols. (2009) realizaron un trabajo encaminado a determinar la validez y fiabilidad de los instrumentos de medida en adherencia terapéutica y concluyeron que ninguna de las pruebas analizadas a través del índice de GraQol alcanzó el $100 \%$, siendo la máxima valoración obtenida de $73 \%$, lo que hace recomendable que siempre sean utilizadas en forma combinada. ${ }^{49}$ De todos los métodos de evaluación de la adherencia los que se han considerado con mayor validez son los basados en el autoinforme, uno de ellos es el test Morisky-Green-Levine. ${ }^{30}$

\section{Redes de apoyo y adherencia al tratamiento}

Las redes de apoyo que influyen en la adherencia, han sido enfocados a fortalecer la educación de pacientes y cuidadores con el fin de identificar de manera más precisa los factores que intervienen en la eficiencia de la aplicación y correcta realización. ${ }^{50}$ La sugerencia es implementar estrategias educativas participativas que faciliten la comprensión del conocimiento ${ }^{51}$ y el desarrollo de habilidades en los pacientes en tratamiento dialítico para garantizar un aprendizaje significativo y permanente..$^{52,53}$ No se ha demostrado que intervenciones aisladas o un conjunto dado de intervenciones sea eficaz en todos los pacientes, enfermedades y entornos. Hay una tendencia en los cuidadores a centrar la energía y esfuerzo para controlar el impacto o efecto negativo más que a solucionar problemas y buscar beneficios para la salud mental y física. Ante esto, los investigadores han sugerido que el apoyo social reduce las consecuencias negativas de los acontecimientos estresantes que se producen en el cuidado de enfermos crónicos. $^{54}$ 
El sistema de salud al cual esté afiliado el paciente con ERC, cuenta como red de apoyo cuando garantiza suplir las necesidades del tratamiento y la oportuna atención. Busca disminuir costos y facilitar la permanencia del paciente en el programa mediante la creación de consultas y protocolos que permiten al paciente y al personal de salud estar mucho más informado acerca del manejo, la técnica a utilizar, el tipo de tratamiento y a su vez responder dudas y garantizar la continuidad del mismo. ${ }^{18}$

\section{Conclusiones}

Con base en la revisión de artículos sobre adherencia en pacientes en DP se puede decir lo siguiente:

La adherencia al tratamiento está relacionada con la capacidad de autocuidado de las personas; como no hay ninguna estrategia que sirva para todos, es necesario el análisis de cada persona y su contexto para definir la más acorde a sus necesidades, sin olvidar que existen factores comunes que orientan al equipo de salud.

Las intervenciones del personal de salud encaminadas a fortalecer la adherencia deben estar basadas en una relación simétrica, respetuosa de las creencias y enfocadas a lograr la voluntad de autocuidarse; deben retroalimentarse y realizar reentrenamientos con el fin de disminuir las complicaciones.

Hay avances en los estudios y herramientas desarrolladas por personal de salud e instituciones que buscan conocer como es la adherencia al tratamiento en condición de cronicidad en DP parámetros clínicos (ganancia de peso interdialítico, episodios de peritonitis) y bioquímicos (hipercalemia, elevación del nitrógeno ureico y la creatinina). Los instrumentos de medición de adherencia que han mostrado mejores resultados son los autoadministrados como el test de Morisky Green, pero con el fin de incluir las dimensiones del ser humano se han diseñado otros como la "escala de adherencia terapéutica para pacientes con enfermedades crónicas, basada en comportamientos explícitos" la cual sería importante validar para pacientes en DP.
Es importante adecuar el horario para los recambios a las necesidades individuales de los pacientes y sus familias, lo cual genera independencia y mayor responsabilidad con el autocuidado, además al no interferir con las labores cotidianas hay mayor bienestar y mejor calidad de vida.

La revisión reportó la utilidad de intervenciones, informativas, educativas, de apoyo familiar y dinámicas de grupo de intervenciones de tipo conductual, para incrementar la adherencia.

La visita domiciliaria es una herramienta muy útil en DP, dado que permite al personal de salud que la realiza, tener conocimiento directo de las condiciones físicas y sociales en que se realiza la DP.

\section{Referencias}

1. Von Mühlen E, Dudel Mayer BL, Ubessi LD, Kirchner RS, Barbosa DA, Fernandes Stumm EM, et al. Análisis de las percepciones de los pacientes con respecto a la actitud del equipo de una unidad de nefrología. Rev. Enfermería Global [serie en Internet]. 2013 Ene [citado 29 Abr 2014]; 12(1): [aprox. 8 p.]. Disponible en: http://revistas.um.es/eglobal/article/view/142031.

2. Rodríguez J. Historia de la diálisis peritoneal [monografía en Internet]. Madrid, España: MedicBlogs; 2011 [citado 29 abr 2014]. Disponible en: http://medicablogs.diariomedico.com/jluisr/historia-de-la-dialisis-peritoneal.

3. Alabart E, Alós B, Barada C, Colli G, González R, Romero A, et al. Enfermedad renal crónica: una epidemia. Med Univer [serie en Internet]. 2009 mar [citado 29 Abr 2014]; 5(1): [aprox. 8 p.]. Disponible en: http://rmu.fcm.uncu.edu.ar/ vol05_01/11/index.php.

4. Gómez RA. Registro colombiano de diálisis y trasplante [monografía en Internet]. Bogotá: Sociedad Colombiana de Nefrología e Hipertensión; 2007 [citado 29 abr 2014]. Disponible en: www.asocolnef.org/userfiles/file/registro_Colombiano.ppt

5. Sanabria M, Muñoz J, Trillos C, Hernández G, Latorre C, Díaz CS et al. Dialysis outcomes in Colombia (DOC) study: A comparison of patient survival on peritoneal dialysis vs hemodialysis in Colombia. Kidney Int Suppl. 2008 Apr;(108):S165-72

6. Ruiz de Alegría, Fernández de Retana, Basabe Barañano N, Fernández Prado E, Baños Baños C, Nogales Rodríguez MA, et al. Calidad de vida y afrontamiento: diferencias entre los pacientes de diálisis peritoneal continua ambulatoria y hemodiálisis hospitalaria. Enferm Clin. 2009;19(2):61-8.

7. Fondo Colombiano de Enfermedades de Alto Costo. Enfermedad renal crónica en Colombia 2008: Resolución 4700 de 2008 cuenta de alto costo. Bogotá: FCEAC; 2010

8. Carballo MR, Ortega N, Lizárraga E, Díaz J. Adherencia individual y familiar al tratamiento de diálisis peritoneal ambulatoria continúa. Rev Enferm Inst Mex Seguro Soc. 2008;16(1): 13-8.

9. OMS. Adherencia a los tratamientos a largo plazo, pruebas para la acción: definición de la adherencia terapéutica [monografía en Internet]. Washington: OMS; 2004 [citado 29 abr 2014]. Disponible en: http://www.paho.org/spanish/ad/dpc/ nc/nc-adherencia.pdf

10. Ortiz PM, Ortiz PE. Psicología de la salud: una clave para comprender el fenómeno de la adherencia terapéutica. Rev Méd Chile [serie en Internet]. 2007 [citado 29 Abr 2014]; 135(5): [aprox. 6 p.]. Disponible en: http://www.scielo.cl/scielo. php?script=sci_arttext\&pid=S0034-98872007000500014

11. Etxeberria A, Rotaeche R, Rico R, Gracia J. Evaluación y síntesis de la evidencia científica terapéutica [monografía en Internet]. Madrid, España: Ministerio de Sanidad, Servicios Sociales e Igualdad; 2008 [citado 29 abr 2014]. Disponible en : http://www.guiasalud.es/emanuales/elaboracion/apartado06/evaluacion.html

12. López AL, Guerrero S. Perspectiva internacional del uso de la teoría general de Orem. Invest Educ Enferm. 2006 sep; 24(2): 90-100. 
13. Hoyos C. Un modelo para investigación documental. Guía teórico-práctica sobre construcción de Estados del Arte con importantes reflexiones sobre la investigación. Medellín: Señal Editora; 2000.

14. Haynes RB, Taylor DW, Sackett DL. Compliance in health care. Baltimore: Johns Hopkins University Press; 1979.

15. Soria R, Vega CZ, Nava C. Escala de adherencia terapéutica para pacientes con enfermedades crónicas, basada en comportamientos explícitos. Altern. psicol [serie en Internet]. 2009 [citado 29 Abr 2014]; 14(20): [aprox. 6 p.]. Disponible en: http://pepsic.bvsalud.org/scielo.php?script=sci_arttext\&pid=S1405-339X20090 $00100008 \& \operatorname{lng}=$ pt\&nrm $=$ iso

16. Nieto C. Diálisis peritoneal enfermería. La enfermera de dialisis peritoneal [monografía en Internet]. Madrid, España: Ministerio de Sanidad, Servicios Sociales e Igualdad; 2008 [citado 29 abr 2014].Disponible en: http://es.scribd.com/ doc/19666891/07

17. Chile. Ministerio de Salud.Guía clínica diálisis peritoneal [monografía en Internet]. Santiago: Minsal; 2010 [citado 29 abr 2014]. Disponible en: http://web.minsal.cl/portal/url/item/99d155829737ee10e04001011e01082b.pdf

18. OMS. $59^{\circ}$ asamblea Mundial de la Salud: actas resumidas e informes de las Comisiones [monografía en Internet]. Ginebra: OMS; 2006 [citado 29 abr 2014]. Disponible en: http://apps.who.int/gb/ebwha/pdf_files/WHA59-REC3/A59RE C3_sp.pdf

19. Colombia. Ministerio de la Protección Social, Fedesalud. Guía para el manejo de la enfermedad Renal Crónica y modelo de prevención y control de la enfermedad renal crónica, basada en la evidencia [monografía en Internet]. Bogotá: Ministerio de la Protección Social; 2007 [citado 29 abr 2014]. Disponible en: http:// www.nacer.udea.edu.co/pdf/libros/guiamps/guiarenal.pdf

20. Venado A. Moreno JA, Rodríguez M, Cervantes M. Insuficiencia Renal Crónica. México: Universidad Nacional Autónoma de México. Unidad de Proyectos Especiales; 2009.

21. Cárdenas A. La ansiedad como factor de riesgo en la adherencia al tratamiento dialítico en pacientes pediátricos con falla renal crónica estadio $\mathrm{V}$. una mirada desde las diferentes terapias de reemplazo renal. Rev Vanguard Psicol. 2011; 2(1):102-13.

22. Malheiro Oliveira P, Arruda S. Percepciones de las personas con insuficiencia renal crónica sobre la calidad de vida. Enfermería Glob. 2012 oct.; 28: 257-75

23. Martínez-Vega AL, Alberto-Bazán ME, Morales-De la Cruz M. Factores favorables para la realización de la Diálisis Peritoneal Continua Ambulatoria en el hogar. Rev. CONAMED. 2010; 15(3):140-46.

24. Rivera A, Montero M, Sandoval R. Desajuste psicológico, calidad de vida y afrontamiento en pacientes diabéticos con insuficiencia renal crónica en diálisis peritoneal. Salud Mental. 2012;35:329-37.

25. Ochando García A, Royuela García C, Hernández Meca ME, Lorenzo Martínez $\mathrm{S}$, Paniagua F. Influencia de la satisfacción de los pacientes de una unidad de diálisis en la adherencia terapéutica. Rev. Soc. Esp. Enferm. Nefrol. 2008; 11(4): 271-76.

26. García - Llana H, Remor E, Selgas R. Adherence to treatment, emotional state and quality of life in patients with end-stage renal disease undergoing dialysis. Psicothema. 2013; 25 (1): 79-86.

27. Santillán Torres Torija C. Diseño, implementación y evaluación de un programa de Intervención Educativa para pacientes con insuficiencia renal Crónica. Rev. latinoam. med. Conducta. 2010; 1(1):37-46.

28. Auer J. Diálisis - Un problema familiar. Tributo personal a los familiares de los pacientes renales. EDTNA/ERCA J. 2002; 28(3):153-57.

29. López J, Sánchez L, Mengibar R, Jiménez S, Marchal M. Aproximación a los cuidados informales de enfermos renales crónicos en el complejo hospitalario de Jaén. Rev Soc Esp Enferm Nefrol. 2007; 10 (2): 127-36.

30. Vélez Vélez E. Representaciones de la insuficiencia renal crónica; afrontamiento y adherencia al tratamiento [tesis en Internet]. Madrid, España: Universidad de Alcalá. Departamento de Psicopedagogía y Educación Física; 2010 [citado 29 abr 2014]. Disponible en: http://dspace.uah.es/dspace/bitstream/handle/10017/9001/ Tesis $\% 20 \%$ 20EVELEZ\%20FInal\%20mayoI10.pdf?sequence $=1$

31. Sánchez JA. La diálisis peritoneal en la planificación integral del tratamiento sustitutivo renal [monografía en Internet]. Madrid, España: Sociedad Española de Nefrología, GADDPE, Fundación Renal AlLCER; 2010 [citado 29 abr 2014]. Disponible en: www.alcer.org

32. BAXTER. Sistemas de DPA HOMECHOICE y HOMECHOICE PRO: guía para el paciente en el hogar. [monografía en Internet]. Deerfield, IL: Baxter; 2010 [citado 29 abr 2014]. Disponible en: http://www.baxter.com/downloads/patients_and_caregivers/products/10.4_FULL_VERSION_SPANISH\%20 PAHG_071963293SPA.pdf

33. Montenegro J, Correa R, Riella M. Tratado de Diálisis peritoneal. Madrid, España: Elsevier; 2009.
34. Vega MG. Conocimientos y habilidades de autocuidado que tienen las familias de pacientes con Diálisis Peritoneal. Desarrollo Científ Enferm [serie en Internet]. 2008 [citado 29 Abr 2014]; 16(4): [aprox. 8 p.]. Disponible en: http://www. index-f.com/dce/16pdf/16-175.pdf

35. NANDA International. Nursing diagnoses: defitions and classification 20092011. Madrid, España: Elsevier; 2010

36. Johnson M, Bulecheck GM, Butcher HK, Dotchterman JM, Maas ML, Swanson E. NANDA,NOC and NIC Linkages: nursing diagnoses, outcomes, and interventions. Philadelphia: Elsevier, Mosby; 2008.

37. Marrón B, Ortiz A, Aguilera A, Remón C, Vega N. Estrategias en la profilaxis y el tratamiento de la malnutrición en diálisis peritoneal: un problema infradiagnosticado. Diál Traspl. 2009; 30(3): 99-9.

38. Prieto MA, Escudero MJ, Suess A, March JC, Ruiz A, Danet A. Análisis de calidad percibida y expectativas de pacientes en el proceso asistencial de diálisis. An. Sist. Sanit. Navar. 2011; 34 (1): 21-31.

39. Instituto nacional de servicios sociales para jubilados y Pensionados. Plan de acción internacional de Madrid sobre el envejecimiento Seguimiento de la declaración de Brasilia [monografía en Internet]. Buenos Aires: PAMI; 2012 [citado 29 abr 2014]. Disponible en: http://www.cepal.org/celade/noticias/paginas/9/46849/ Argentina_INSSJP.pdf

40. Torres MP, Ballesteros E, Sánchez PD. Programas e intervenciones de apoyo a los cuidadores informales en España. Gerokomos [serie en Internet]. 2008 mar [citado 29 Abr 2014]; 19(1): [aprox. 8 p.]. Disponible en: http://dx.doi. org/10.4321/S1134-928X2008000100002

41. Pegoraro M. Experiencia de la enfermería italiana en un programa de CAPD Enferm Nefrol. 1996:1996(1):24-6.

42. Cramer JA, Mattson RH, Prevey ML, Scheyer RD, Ouellette VL. How often is medication taken as prescribed? A novel assessment technique. JAMA. 1989 Jun 9;261(22):3273-7.

43. OMS. Adherencia a los tratamientos a largo plazo. Pruebas para la acción [monografía en Internet]. Washington: OMS; 2004 [citado 29 abr 2014]. Disponible en: http://www.paho.org/spanish/ad/dpc/nc/nc-adherencia.pdf.

44. Carrillo Algarra AJ, Díaz FJ. Capacidad de autocuidado de los pacientes en diálisis peritoneal. Enferm. glob. [serie en Internet]. 2013 abr [citado 29 abr 2014]; 12(30): [aprox. 8 p.]. Disponible en: http://scielo.isciii.es/scielo.php?pid=S1695$61412013000200004 \&$ script=sci arttext

45. You +1 'd this publicly. UndXXXII Congreso Anual de la Sociedad Española de Diálisis y Trasplante. Dial Traspl. 2010;31(3):106-15.

46. De Sousa Pacheco G, Dos Santos I, Bregman R. Clientes con enfermedad renal crónica: evaluación de enfermería sobre la competencia para el autocuidado. Esc Anna Nery R Enferm. 2007 mar; 11 (1): 44 - 51.

47. Nau DP, Steinke DT, Williams LK, Austin R, Lafata JE, Divine G, Pladeval M. Adherence analysis using visual analog scale versus claims-based estimation. Ann Pharmacother. 2007 Nov;41(11):1792-7.

48. Corugedo Rodríguez MC, Martín Alfonso Libertad, Bayarre Vea Héctor. Adherencia terapéutica en pacientes con hipertensión arterial del Policlínico Universitario "Manuel Fajardo" en el Municipio Cruces, Cienfuegos, 2009. Rev Cubana Med Gen Integr [serie en Internet]. 2011 Dic [citado 29 abr 2014 ]; 27(4): [aprox. 8 p.]. Disponible en: http://scielo.sld.cu/scielo.php?script=sci_ arttext\&pid=S0864-21252011000400009\&lng=es

49. García-Fernández MN; De Cabo-Laso A, Nogales-Carpintero JI, Otero-López MC, De la Vega-Carnicero J, Herrero-García R, et al. Validez y fiabilidad de los instrumentos de medida en adherencia terapéutica: detectar los no cumplidores [monografía en Internet]. ].Castilla y León: Junta de Castilla y León. Consejería de Sanidad; 2009 [citado 29 abr 2014]. Disponible en: http://www.saludcastillayleon.es/profesionales/es/invesalud/banco-evidencias-cuidados.

50. Montenegro Martínez J. Peritonitis e infecciones del catéter en la diálisis peritoneal [monografía en Internet]. ]. Madrid, España: Sociedad Española de Nefrología; 2010 [citado 29 abr 2014]. Disponible en: http://nefrologiadigital. revistanefrologia.com/modules.php?name=libro\&op=viewCap\&idpublication=1 \&idedition $=13 \&$ idcapitulo $=88$

51. Teixidó J, Tarrats L, Arias N, Cosculluela A. Cuestionario de sobrecarga de cuidadores de pacientes de diálisis peritoneal. Unidad de Diálisis Peritoneal. Nefrologia 2006;26(1):74-83.

52. Tapia Yañez T, Muñoz Montes A, Mendez Durán A. La consulta de enfermeria, propuesta de mejora en los programas de dialisis peritoneal. Rev Enferm Inst Mex Seguro Soc. 2010; 18 (3): 159-62.

53. Ponz Clemente E, Martínez Ocaña JC, Marquina Parra D, Blasco Cabañas C, Grau Pueyo G, Mañé Buixó N, García García M. Analysis of psychological factors influencing peritoneal dialysis selection. Nefrologia. 2010;30(2):195-201.

54. Vega Angarita OM, González Escobar DS. Apoyo social: elemento clave en el afrontamiento de la enfermedad crónica social. Enferm. Glob [serie en Internet]. 2009 jun [citado 29 abr 2014]; 16: [aprox. 11 p.]. Disponible en: http://scielo. isciii.es/pdf/eg/n16/revision2.pdf 